Supporting Information for

\title{
In Situ Monitoring of the Seeding and Growth of Silver Metal-Organic Nanotubes by Liquid-Cell Transmission Electron Microscopy
}

Karthikeyan Gnanasekaran, ${ }^{a}$ Kristina M. Vailonis, ${ }^{b}$ David M. Jenkins, ${ }^{b,{ }^{*}}$ Nathan C. Gianneschi ${ }^{a},{ }^{*}$

a Department of Chemistry, Department of Materials Science \& Engineering, Department of Biomedical Engineering, Department of Pharmacology, International Institute for Nanotechnology, Simpson Querrey Institute, Chemistry of Life Processes Institute, Northwestern University, Evanston, Illinois 60208, United States.

${ }^{b}$ Department of Chemistry, University of Tennessee, Knoxville, Tennessee 37996, United States.

Table of Contents

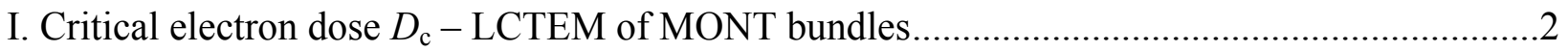

II. LCTEM of growth of highly anisotropic MONT bundles ..........................................................

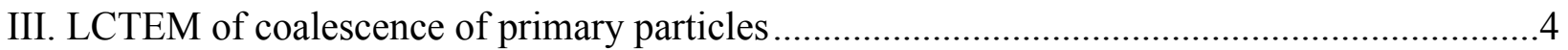

IV. Liquid-thickness measurements ........................................................................................

V. Image analysis - segmentation and quantification of MONT bundles......................................

VI. MONT growth kinetics measurements...........................................................................

VII. Post-mortem EDS of MONT bundles grown within liquid-cell ............................................

VIII. TEM of MONT growth in bulk synthesis.......................................................................10

IX. AFM of MONT grown in bulk synthesis ........................................................................1

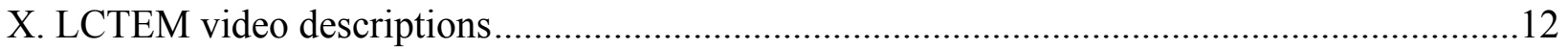

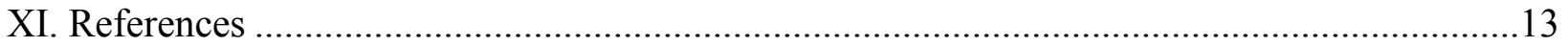




\section{Critical electron dose $D_{c}-$ LCTEM of MONT bundles}

Success in LCTEM experiments on MONT growth require that we minimize the effect of electron beam induced effects and destruction of growing MONT bundles. ${ }^{1,2}$ Kinetic modelling and empirical observation have shown that the electron beam can induce metal nanoparticle nucleation and growth from solutions of metal salts by reduction, with their size increasing monotonically with time through monomer addition. ${ }^{3,4}$ Therefore, the first step was to confirm imaging could be performed without inhibiting or damaging MONT formation by radiolysis or other beam induced effects. ${ }^{4-6}$ We performed MONT growth within the liquid cell without exposure to the electron beam. This was achieved by flowing $30 \mathrm{mg}$ of $\mathrm{AgNO}_{3}$ in $3 \mathrm{~mL}$ of deionized water and $14 \mathrm{mg}$ of $\mathbf{L 1}$ ligand in $3 \mathrm{~mL}$ of NMP at a rate of $1 \mu \mathrm{L} \cdot \mathrm{min}^{-1}$ inside separate tubes, meeting inside the cell (Figure S1). This was done simultaneously into the liquid cell, followed by heating of the liquid-cell to $85^{\circ} \mathrm{C}$ for 15 minutes. The reaction was followed by imaging and hence irradiating the fully-grown MONT to determine the critical electron dose that initiates the destruction of MONT bundles (Figure S2). We identified the structural damage to the MONTs as the cumulative doses exceeded $70 \mathrm{e}^{-} \AA^{-2}$ acquired with an electron flux of $0.36 \mathrm{e}^{-} \AA^{-}$ ${ }^{2} \mathrm{~S}^{-1}$ (Movie S1).
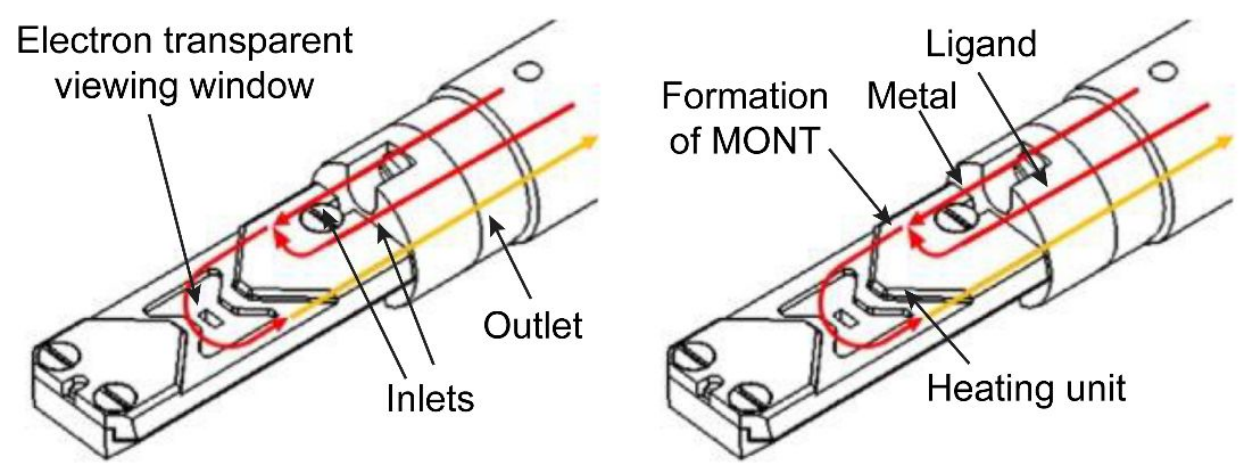

Figure S1. Schematic illustration of flow of reactants in Protochips Poseidon Select ${ }^{\mathrm{TM}}$ Liquidcell TEM holder. 

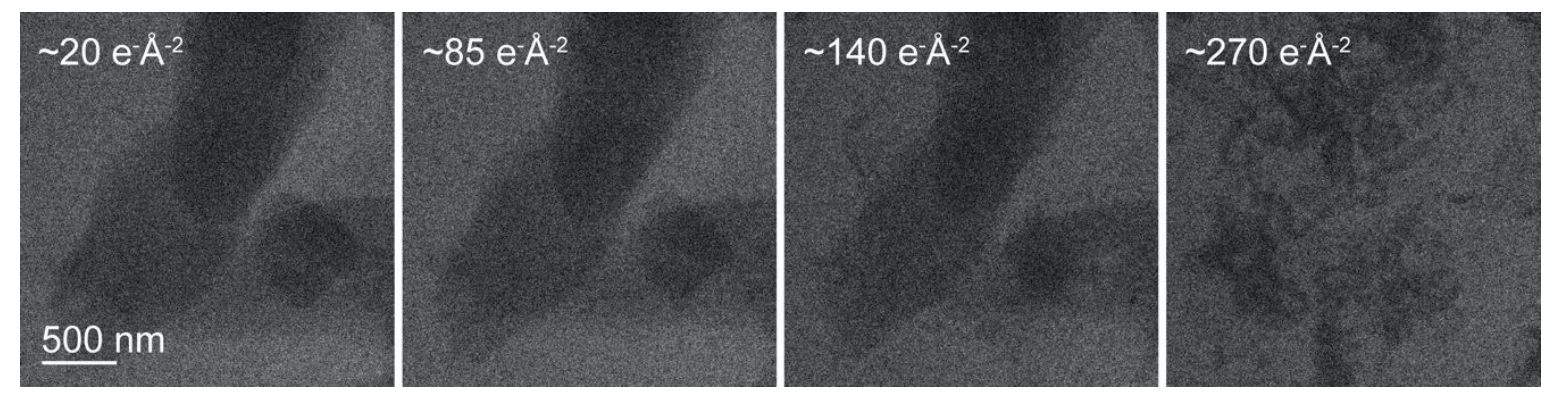

Figure S2. Snapshots of the disintegration of MONT bundles at given cumulative doses, during prolonged exposure to the electron beam acquired with an electron flux of $0.36 \mathrm{e}^{-} \AA^{-2} \mathrm{~s}^{-1}$ (see Movie S1).

\section{LCTEM of growth of highly anisotropic MONT bundles}

Highly anisotropic MONT bundles are formed from 3:1 AgNO3:L1 reaction: $\mathrm{AgNO}_{3}(30$ $\mathrm{mg}, 0.177 \mathrm{mmol}$ ) was dispersed in $6 \mathrm{~mL}$ of deionized water and separately, L1 (14 mg, 0.059 mmol) was dispersed in $6 \mathrm{~mL}$ of NMP. NB: Here the reactants were diluted by $100 \%$ in relation to other experiments.
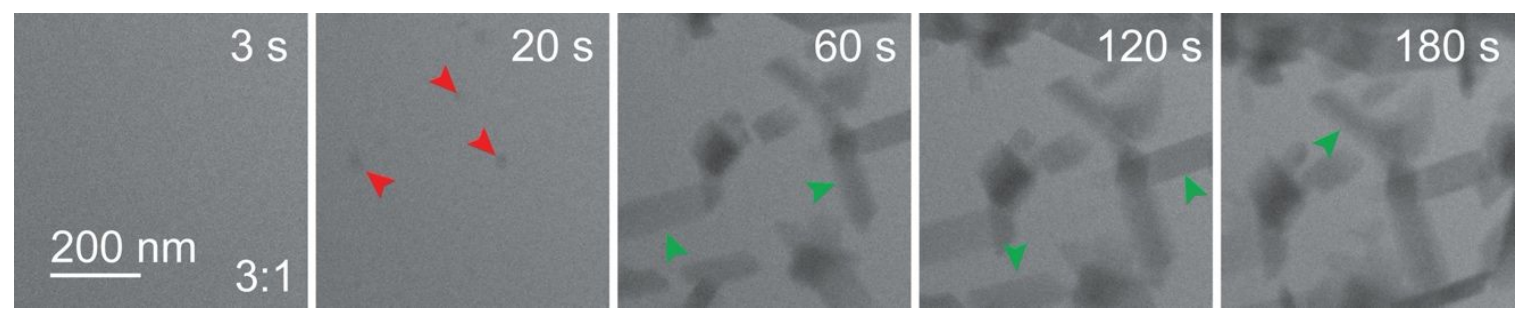

Figure S3. LCTEM snapshots of growth of MONT bundles at 3:1 AgNO3:L1 acquired with an electron flux of $0.2 \mathrm{e}^{-} \AA^{-2} \mathrm{~s}^{-1}$ (Movie S5). Red arrows represent the faceted particles and green arrows represent the MONT bundles. 
III. LCTEM of coalescence of primary particles

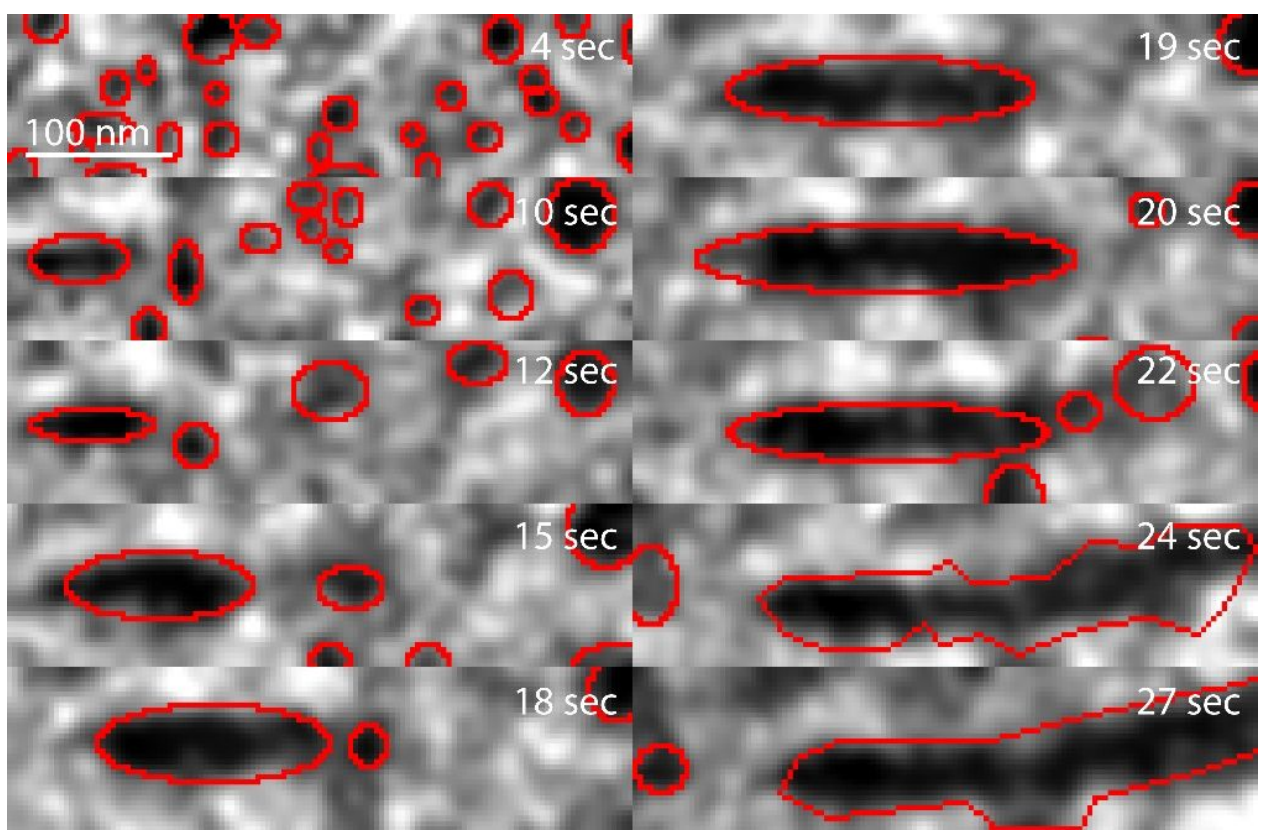

Figure S4. Magnified snapshots of Movie S9 reveal the coalescence of primary particles to form elongated rod-like morphologies of the silver MONT (6:1 $\left.\mathrm{AgNO}_{3}: \mathbf{L 1}\right)$. 


\section{Liquid-thickness measurements}

Liquid thickness was measured based on elastic mean free path of the electrons transmitting through the liquid. ${ }^{7}$ Here, we approximated the major molecular constituent of the liquid sample to solvent (i.e. water and NMP) and calculated its thickness sandwiched between $2 \operatorname{SiN}_{x}$ membranes.

The elastic $\left(\sigma_{\mathrm{el}}\right)$ cross-section of the electrons transmitting through the liquid is given by

$$
\sigma_{\mathrm{el}}=\frac{1.5 \times 10^{-6} Z^{\frac{3}{2}}}{\beta^{2}}\left[1-\frac{0.23 Z}{137 \beta}\right]
$$

where $\beta$ is the ratio between the velocity of an electron and light, $Z$ is the atomic number. Hence, the total elastic scattering cross-sections: $\mathrm{H}_{2} \mathrm{O}=6.03 \mathrm{E}-05 \mathrm{~nm}^{2} ; \mathrm{NMP}=3.03 \mathrm{E}-04 \mathrm{~nm}^{2} ; \mathrm{Si}_{3} \mathrm{~N}_{4}=$ $5.61 \mathrm{E}-04 \mathrm{~nm}^{2}$.

The elastic mean free path of the electron $\left(\Lambda_{\mathrm{el}}\right)$ is related to the molar mass $\left(M_{\mathrm{w}}\right)$, density $\rho$, and the total elastic cross-section $\left(\sigma_{\mathrm{el}}\right)$ as

$$
\Lambda_{\mathrm{el}}=\frac{M_{w}}{\rho N_{\mathrm{A}} \sigma_{\mathrm{el}}}
$$

where $N_{\mathrm{A}}$ is Avogadro's number. The elastic mean free path: $\mathrm{H}_{2} \mathrm{O}=496.27 \mathrm{~nm}$; NMP $=527.67$ $\mathrm{nm} ; \mathrm{Si}_{3} \mathrm{~N}_{4}=131.08 \mathrm{~nm}$. Mean free path of electrons in liquid-cell sample is measured based on the volume fraction of $\mathrm{H}_{2} \mathrm{O}$ and NMP. As we flow-in both the solvents at the same rate, we assume $50-50$ vol. $\%$ of $\mathrm{H}_{2} \mathrm{O}$ and NMP.

$$
\frac{1}{\Lambda}=\frac{0.5}{\Lambda_{H_{2} O}}+\frac{0.5}{\Lambda_{N M P}}
$$

Mean free path, $\Lambda=255.74 \mathrm{~nm}$.

Liquid thickness is related to the elastic mean free path of the electron and the intensity of the electron beam as 


$$
I=I_{0} \mathrm{e}^{-x / \Lambda}
$$

where $I$ is the intensity of the transmitted beam through the sample, $I_{0}$ is the intensity of the beam without sample, $x$ is the liquid thickness, and $\Lambda$ is the elastic mean free path of electron. Note: these intensities were measured by acquiring images during the alignment phase, and immediately after loading the liquid-cell.

$$
x=\left(\ln \left(\frac{I_{S i N}}{I_{0}}\right)-\ln \left(\frac{I_{S i N}+l i q}{I_{0}}\right)\right) \times \Lambda
$$

The measured liquid thicknesses were relatively consistent. Liquid thicknesses of some of the reported movies are: Movie-S01: 323.19 nm; Movie-S02: 368.38 nm; Movie-S04: 341.34 nm; Movie-S05: $353.56 \mathrm{~nm}$; Movie-S06: $379.78 \mathrm{~nm}$. 


\section{Image analysis - segmentation and quantification of MONT bundles}

To quantify the growth parameters of MONT bundles, the acquired time series were segmented frame-by-frame (Figure S4A). First, the acquired time series was median filtered (2x2x5 elliptical) to remove salt and pepper noise. This was followed by Gaussian filters with sigma of 50 or more, and the background was subtracted (Figure S4B). The data was 'Otsu' thresholded to generate binary images where MONTs were set as 1 and rest as 0 (Figure S4C). To further remove the noise, objects smaller than $100 \mathrm{~nm}^{2}$ in area were removed (Figure S4D).
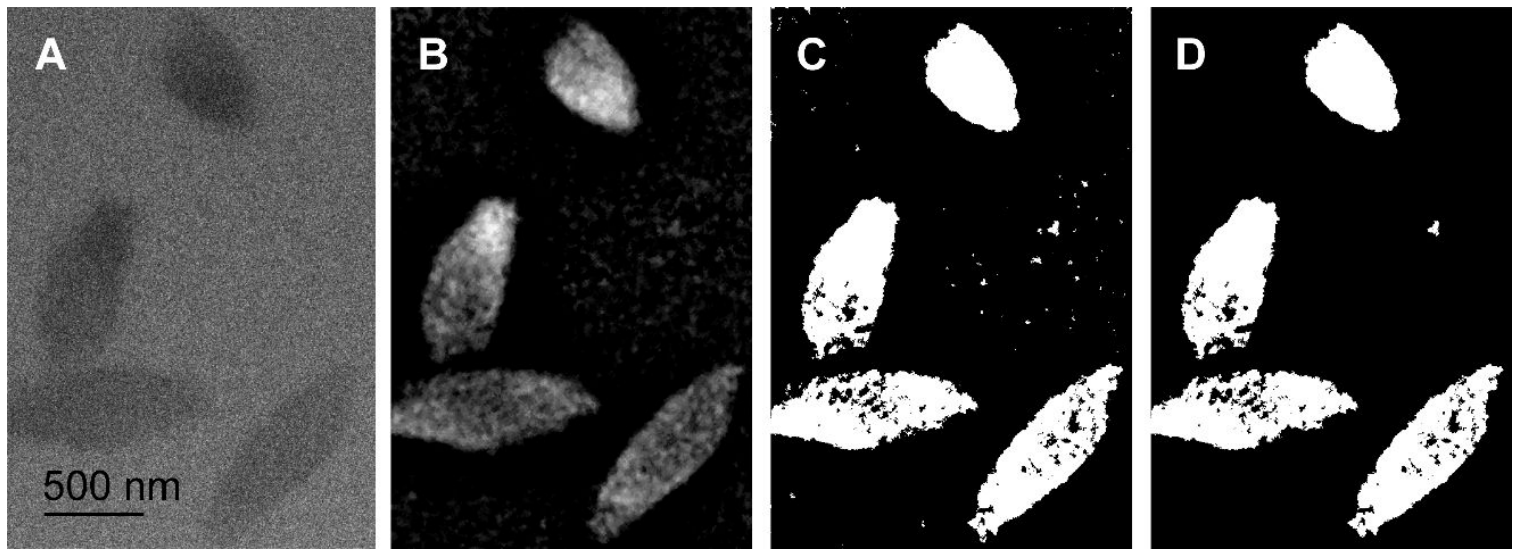

Figure S5. Image analysis performed to obtain the sizes of growing MONT bundles as a function of time. 


\section{MONT growth kinetics measurements}

First, average area (in $\mathrm{nm}^{2}$ ) was quantified for each frame from the binarized data (Figure S5). The average size $\langle S\rangle$ of MONT bundles at each frame is represented by the square root of the area in $\mathrm{nm}$. The growth rate of MONT bundles is measured by fitting the size $\langle S\rangle$ vs. time $t$ (in s) data to power law: $\langle S\rangle=G t^{n}+\mathrm{c}$ where $G$ represents the growth rate in linear regime (in nm.s $\mathrm{s}^{-1}$ ), c is the intercept, $n$ is the growth exponent that reveals whether the growth process is predominately controlled by diffusion (when $n \sim 1 / 2$ ) or reaction (when $n \sim 1$ ) as proposed by the Lifshitz-Slyozov-Wagner (LSW) model. ${ }^{8-10}$

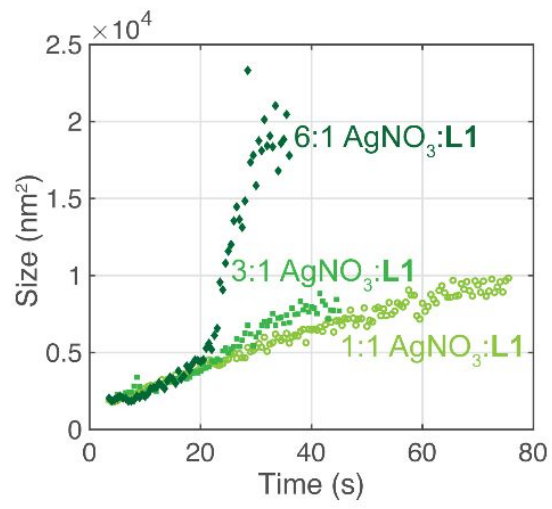

Figure S6. Size of MONT bundles as a function of time for various reaction conditions.

The following fit parameters were obtained for our system:

$1: 1 \mathrm{AgNO}_{3}: \mathbf{L 1}:$

$t<21 \rightarrow G=1.27, n=1, \mathrm{c}=38.03 ; t>21 \mathrm{~s} \rightarrow G=8.14, n=0.5, \mathrm{c}=28.13$

$3: 1 \mathrm{AgNO}_{3}: \mathbf{L 1}:$

$0<t<45 \rightarrow G=1.25, n=1, \mathrm{c}=39.24$

6:1 $\mathrm{AgNO}_{3}: \mathbf{L 1}:$

$t<20 \rightarrow G=1.51, n=1, \mathrm{c}=34.91 ; 21<t<30 \rightarrow G=7.68, n=1, \mathrm{c}=-89.3$ 


\section{Post-mortem EDS of MONT bundles grown within liquid-cell}

Following growth experiments by LCTEM, the chips were opened carefully and washed gently with distilled water to remove excess reactants and solvent. The chips were then placed on the standard TEM holder and analyzed by EDS and diffraction.

A

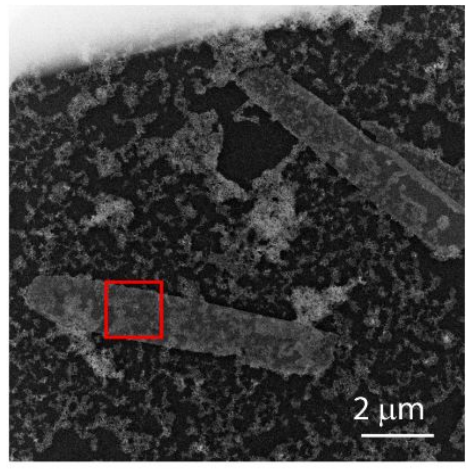

B

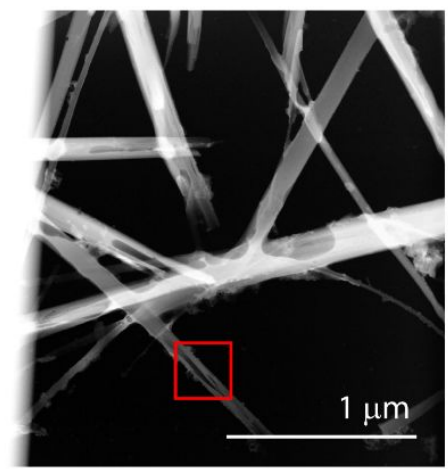

C

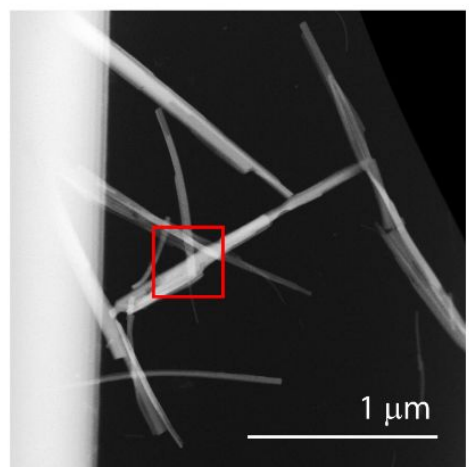

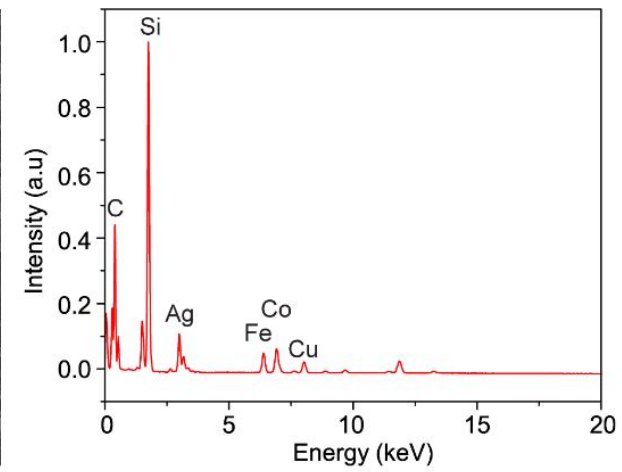
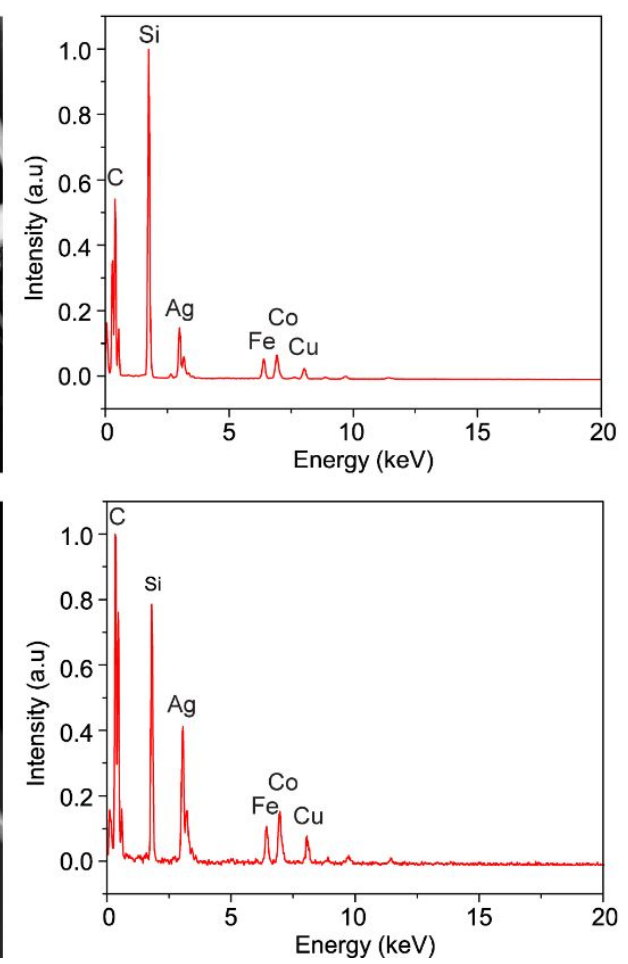
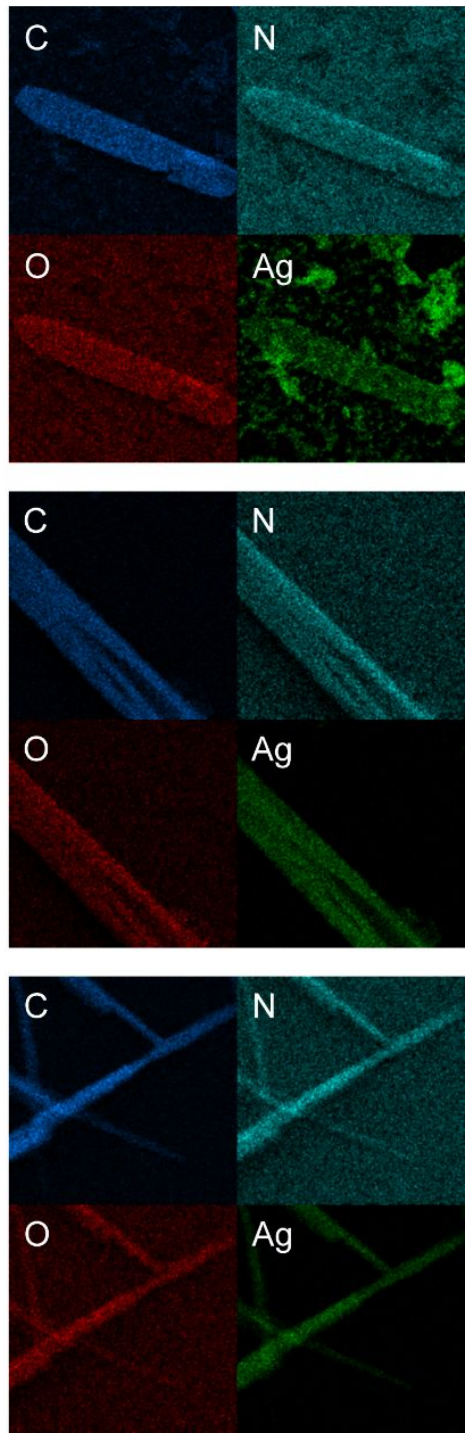

Figure S7. Post-mortem analysis of MONT bundles grown within the liquid-cell. HAADF-STEM images, EDS spectra and elemental mapping of MONT primary particles and MONT bundles formed within the liquid-cell. A) 1:1 $\mathrm{AgNO}_{3}: \mathbf{L 1}$; B) $3: 1 \mathrm{AgNO}_{3}: \mathbf{L 1}$; C) $6: 1 \mathrm{AgNO}_{3}: \mathbf{L 1}$. 
VIII. TEM of MONT growth in bulk synthesis
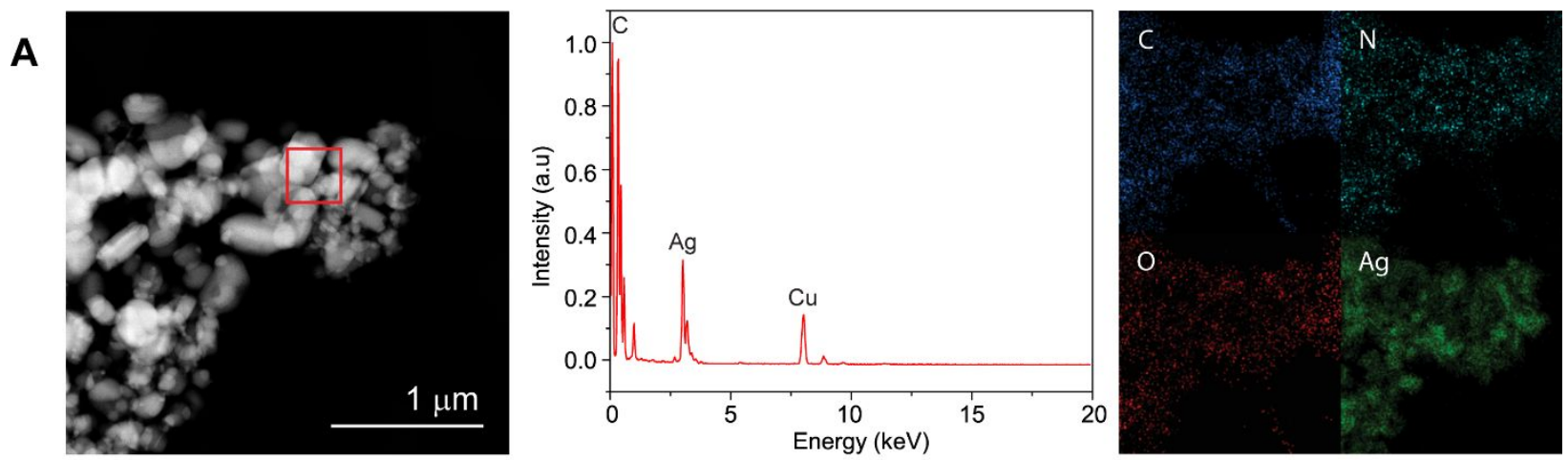

B
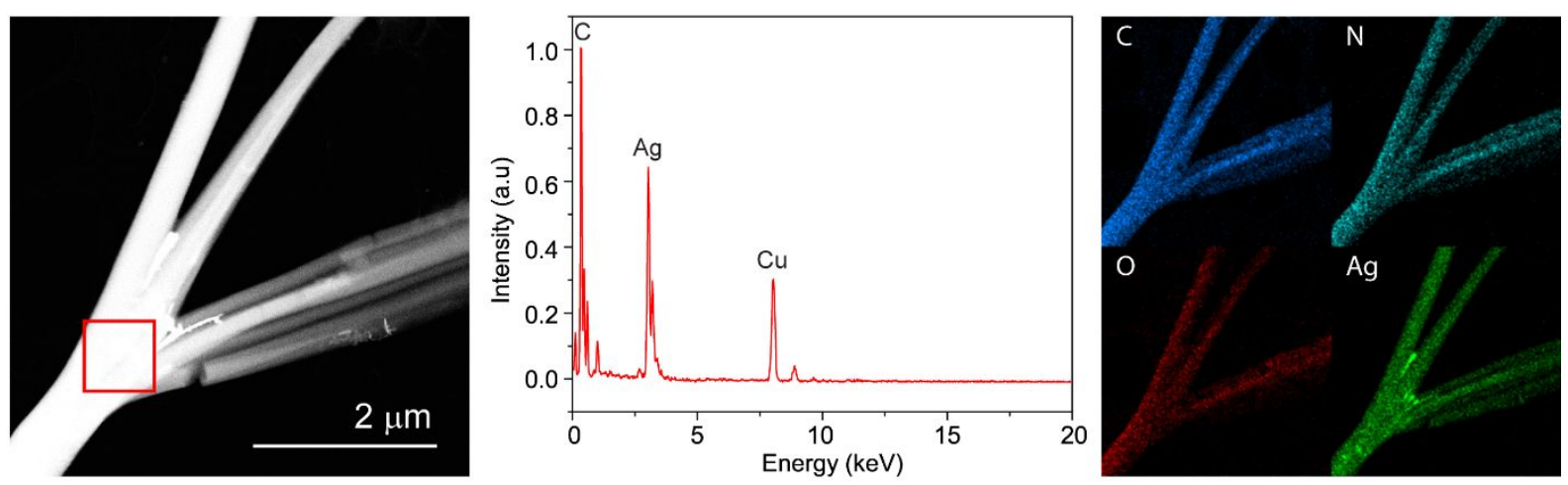

C
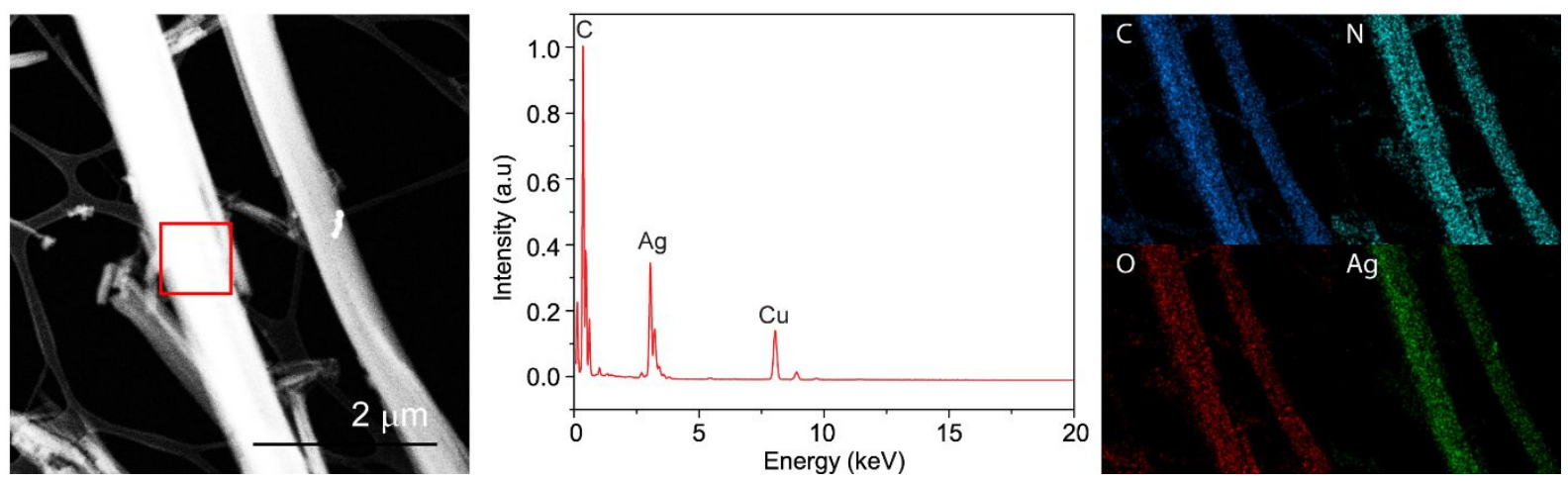

Figure S8. HAADF-STEM images, EDS spectra, and elemental mapping of MONT primary particles and MONT bundles formed in bulk synthesis. A) 1:1 $\mathrm{AgNO}_{3}: \mathbf{L 1}$; B) 3:1 $\mathrm{AgNO}_{3}: \mathbf{L 1}$; C) 6:1 $\mathrm{AgNO}_{3}: \mathbf{L 1}$. 


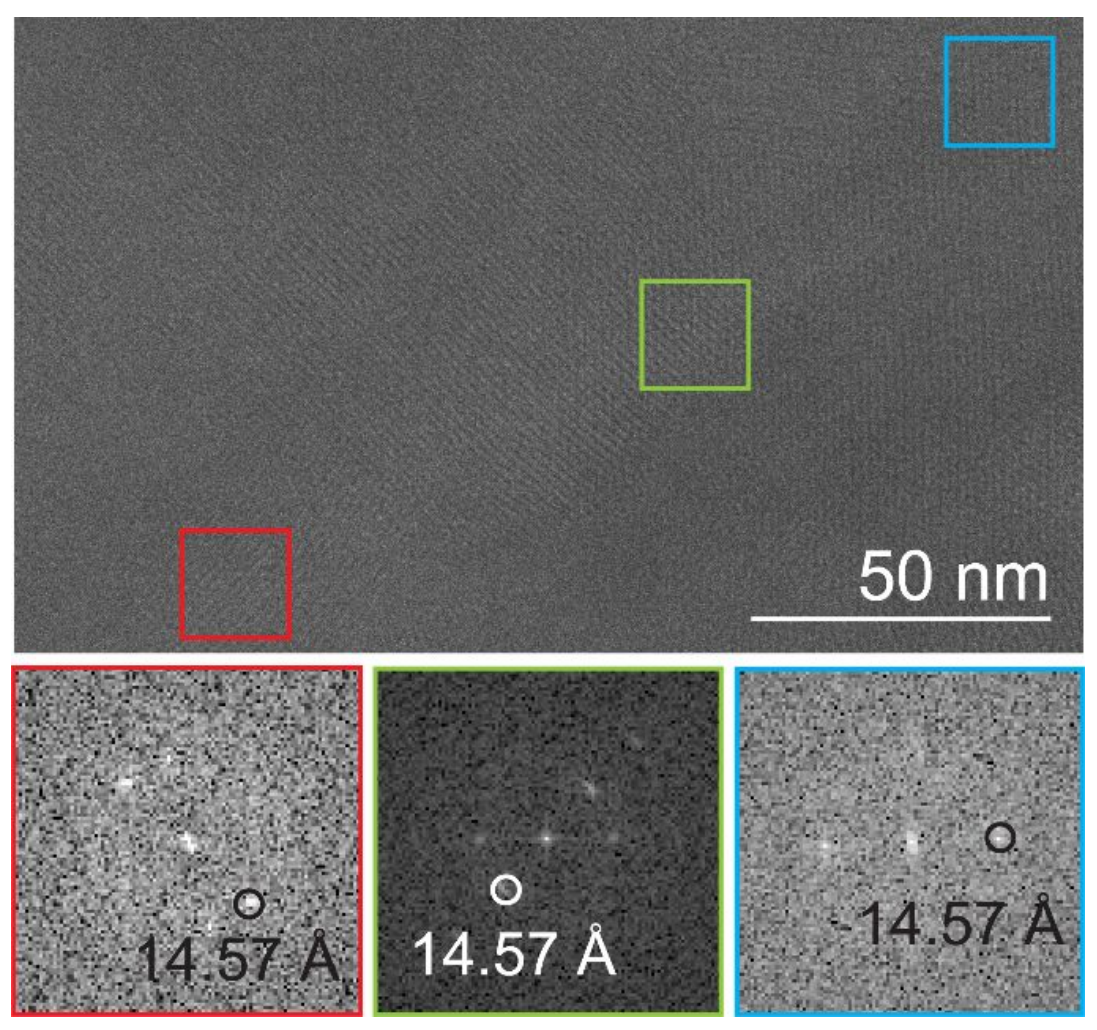

Figure S9. HRTEM image and the corresponding FFT illustrates the aggregation of several MONT bundles.

\section{AFM of MONT grown in bulk synthesis}
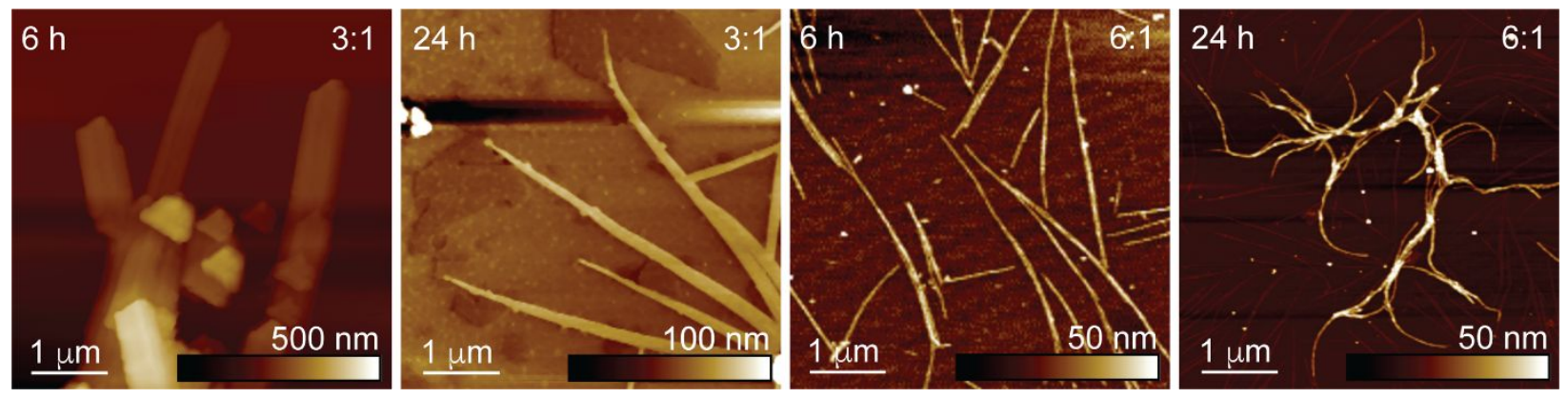

Figure S10. AFM analysis of MONT bundles after 6 hours and 24 hours of reaction in bulk at various ratios (shown in top right corner) of $\mathrm{AgNO}_{3}: \mathbf{L 1}$. 


\section{LCTEM video descriptions}

Movie S01: Beam damage threshold measurement. LCTEM of 3:1 AgNO3:L1 MONT bundles. Data acquired with an electron flux of $0.36 \mathrm{e}^{-} \AA^{-2} \mathrm{~s}^{-1}$.

Movie S02: LCTEM of 1:1 AgNO3:L1 shows the formation of primary particles. Data acquired with an electron flux of $0.36 \mathrm{e}^{-} \AA^{-2} \mathrm{~s}^{-1}$.

Movie S03: Stroboscopic LCTEM acquisition of 1:1 AgNO3:L1. MONT nanotubes are formed on the surface of primary particles. Data acquired with an electron flux of $0.36 \mathrm{e}^{-} \AA^{-2} \mathrm{~s}^{-1}$.

Movie S04: LCTEM of 3:1 AgNO3:L1 - MONT bundles are formed along with primary particles. Data acquired with an electron flux of $0.36 \mathrm{e}^{-} \AA^{-2} \mathrm{~s}^{-1}$.

Movie S05: LCTEM of 3:1 AgNO3:L1 - Data acquired using a Gatan K3-IS camera with an electron flux to $0.2 \mathrm{e}^{-} \AA^{-2} \mathrm{~s}^{-1}$, which allowed us to capture the growth of highly elongated MONT bundles over $\sim 3$ min of reaction.

Movie S06: LCTEM of 6:1 AgNO3:L1 shows the formation of highly anisotropic MONT bundles. Data acquired with an electron flux of $0.36 \mathrm{e}^{-} \AA^{-2} \mathrm{~s}^{-1}$.

Movie S07: LCTEM of 3:1 AgNO3:L1 illustrates the gradual growth of MONT bundles.

Movie S08: LCTEM of 3:1 AgNO3:L1 shows the rapid growth of anisotropic MONT bundles on the surface of the initially formed primary particles.

Movie S09: LCTEM of 6:1 AgNO3:L1 shows the attachment of primary particles to the growing anisotropic MONT bundles. Total time $\sim 25 \mathrm{~s}$. 


\section{References}

1. Vailonis, K. M.; Gnanasekaran, K.; Powers, X. B.; Gianneschi, N. C.; Jenkins, D. M., Elucidating the Growth of Metal-Organic Nanotubes Combining Isoreticular Synthesis with Liquid-Cell Transmission Electron Microscopy. J. Am. Chem. Soc. 2019, 141, 10177-10182.

2. Lyu, J.; Gong, X.; Lee, S. J.; Gnanasekaran, K.; Zhang, X.; Wasson, M. C.; Wang, X.; Bai, P.; Guo, X.; Gianneschi, N. C.; Farha, O. K., Phase Transitions in Metal-Organic Frameworks Directly Monitored through In Situ Variable Temperature Liquid-Cell Transmission Electron Microscopy and In Situ X-Ray Diffraction. J. Am. Chem. Soc. 2020, 142, 4609-4615.

3. Woehl, T. J.; Evans, J. E.; Arslan, I.; Ristenpart, W. D.; Browning, N. D., Direct In Situ Determination of the Mechanisms Controlling Nanoparticle Nucleation and Growth. ACS Nano 2012, 6, 8599-8610.

4. Woehl, T. J.; Abellan, P., Defining the Radiation Chemistry during Liquid Cell Electron Microscopy to Enable Visualization of Nanomaterial Growth and Degradation Dynamics. J. Microsc. 2017, 265, 135-147.

5. Woehl, T. J.; Jungjohann, K. L.; Evans, J. E.; Arslan, I.; Ristenpart, W. D.; Browning, N. D., Experimental Procedures to Mitigate Electron Beam Induced Artifacts during In Situ Fluid Imaging of Nanomaterials. Ultramicroscopy 2013, 127, 53-63.

6. Moser, T. H.; Mehta, H.; Park, C.; Kelly, R. T.; Shokuhfar, T.; Evans, J. E., The Role of Electron Irradiation History in Liquid Cell Transmission Electron Microscopy. Sci. Adv. 2018, 4, eaaq1202.

7. Reimer, L.; Kohl, H., Transmission Electron Microscopy - Physics of Image Formation, Fifth Edition; Springer Series in Optical Sciences, Springer: New York, 2008; 36, pp 590.

8. Viswanatha, R.; Sarma, D. D., Growth of Nanocrystals in Solution. In Nanomaterials Chemistry: Recent Developments and New Directions; Rao, C. N. R., Muller, A. M., Cheetham, A. K., Eds.; Wiley-VCH Verlag: Weinheim, Germany, 2007; pp 139-170. 
9. Wagner, C., Theory of Precipitate Change by Redissolution. Z. Elektrochem. 1961, 65, $581-591$.

10. Lifshitz, I. M.; Slyozov, V. V., The Kinetics of Precipitation from Supersaturated Solid Solutions. J. Phys. Chem. Solids 1961, 19, 35-50. 